\title{
Reproducing Kernel in Krein Spaces
}

\author{
OSMIN FERRER $^{1}$, DIEGO CARRILLO ${ }^{2}$, ARNALDO DE LA BARRERA $^{3}$ \\ ${ }^{1}$ Department of Mathematics, Sucre University, Sincelejo, COLOMBIA \\ ${ }^{2}$ Basic Sciences Department, Caribbean University Corporation, Sincelejo, COLOMBIA \\ ${ }^{3}$ Department of Mathematics, University of Pamplona, Pamplona, COLOMBIA
}

\begin{abstract}
This article describes a new form to introduce a reproducing kernel for a Krein space based on orthogonal projectors enabling to describe the kernel of a Krein space as the difference between the kernel of definite positive subspace and the kernel of definite negative subspace corresponding to kernel of the associated Hilbert space. As application, the authors obtain some basic properties of both kernels for Krein spaces and exhibit that each kernel is uniquely determined by the Krein space given. The methods and results employed generalize the notion of reproducing kernel given in Hilbert spaces to the context of spaces endowed with indefinite metric.
\end{abstract}

Key-Words: - Hilbert space, Krein space, reproducing kernel, indefinite metric.

Received: March 21, 2021. Revised: November 17, 2021. Accepted: December 22, 2021. Published: January 11, 2022.

\section{Introduction}

A Hilbert space endowed with a function such that reproduces every element of space through the internal product is called a Hilbert space with reproducing kernel or a reproducing kernel Hilbert space. The theory of these spaces plays an important role in many branches of mathematics such that functional analysis, harmonic analysis, operator theory and quantum mechanics [1], [2] and [3]. The concept of reproducing kernel was used first at the early twentieth century by Zaremba [4]. The same author studied other functions that satisfy a property of reproducing in the theory of integral equations, called definite positive kernels [5]. Later, Bergman [6] introduced reproducing kernel in one and several variables for the class of harmonic functions and the class of analytic functions, called kernel functions. After them many authors have studied the same idea as Szego [7], even in recent years there has been considerable interest in studying the reproducing properties in functional analysis, harmonic analysis, operator theory, quantum mechanics, among other fields; which can be seen in [8], [9], [10] and [11]. In [12] a reproducing kernel structure was introduced for Hilbert spaces with functions where the differences of point evaluations are limited and in [13] they used the reproducing kernel to study model spaces generated by certain types of singular inner functions. Motivated by these researches, in this paper we extend the notion of reproducing kernel to more general spaces such that Krein spaces [14], [15], [16] and [17]. The principal research method used in this work is the hypothetical- deductive method. In this work we characterize the existence of a reproducing kernel for a Krein space through two important Hilbert subspaces, called definite positive subspace and definite negative subspace. By using these subspaces, we describe the kernel of a Krein space as the difference between the kernel of definite positive subspace and the kernel of definite negative subspace, while the sum of these subspaces corresponds to kernel of the associated Hilbert space. Also, we prove some basic properties of both kernels for spaces with indefinite metric and we exhibit that each kernel is uniquely determined by the Krein space given. From this, we generalize the notion of reproducing kernel given in Hilbert spaces to the context of spaces endowed with indefinite metric. The results of our research can be potentially important for mathematicians who working in areas such that functional analysis, harmonic analysis, operator theory and quantum mechanics and others affine areas.

\section{Problem Formulation}

Starting from the reproducing kernel defined in Hilbert spaces, the generalization of this concept was pursued in spaces with undefined metrics, more specifically in Krein spaces. It was even sought to verify some basic properties for both kernel and in turn show that each kernels is uniquely determined by the given Krein space. 


\subsection{Kernels on Hilbert Spaces}

Definition 2.1. Let $\mathcal{H}$ be a Hilbert space of complex-valued functions on a set $\mathrm{X}$. Given $\mathrm{f}, \mathrm{g} \in \mathcal{H}$, we denoted by $(\mathrm{f}, \mathrm{g})$ the internal product of $f$ and g. The complex-valued function $\mathcal{N}: \mathrm{X} \times \mathrm{X} \rightarrow \mathbb{C}$ denoted by $\mathcal{N}_{\mathrm{y}}(\mathrm{x}):=\mathcal{N}(\mathrm{x}, \mathrm{y})$ is called a reproducing kernel for $\left(\mathcal{H}, \psi_{s}\right)$ ), if this function satisfies:

i) For each $y \in \mathrm{X}$ the function $\mathcal{N}_{\mathrm{y}}$ defined by

$$
\mathcal{N}_{\mathrm{y}}(\mathrm{x}) \mathrm{x}=\mathcal{N}(\mathrm{x}, \mathrm{y}) \text { is an element of } \mathcal{H}
$$

ii) For each $\mathrm{x} \in \mathrm{X}$ and $\mathrm{f} \in \mathcal{H}$, the reproducing

$$
\text { property } \left.\mathrm{f}(\mathrm{x})=\left\langle\mathrm{f}, \mathcal{N}_{\mathrm{x}}\right\rangle\right) \text { is fulfilled. }
$$

Proposition 2.2. Let $\mathcal{H}$ be a Hilbert space with reproducing kernel $\mathcal{N}: \mathrm{X} \times \mathrm{X} \rightarrow \mathbb{C}, \quad$ then $\left\|\mathcal{N}_{\mathrm{x}}\right\|^{2}=\mathcal{N}(\mathrm{x}, \mathrm{x}), \quad \forall \mathrm{x} \in \mathrm{X}$

A Hilbert space $\mathcal{H}$ of complex-valued functions on a set $\mathrm{X}$ is called a Hilbert space with a reproducing kernel (often abbreviated EHNR) if there exists a reproducing kernel $\mathcal{N}$ associated to $\mathcal{H}$.

Proposition 2.3. Let $\mathcal{H}$ be a Hilbert space with reproducing kernel $\mathcal{N}$, then $\mathcal{N}$ satisfies the following properties:

i) $\quad \mathcal{N}(\mathrm{x}, \mathrm{x}) \geq 0$

ii) $\mathcal{N}(\mathrm{x}, \mathrm{y})=\overline{\mathcal{N}(\mathrm{y}, \mathrm{x})}$

iii) $|\mathcal{N}(\mathrm{x}, \mathrm{y})|^{2} \leq \mathcal{N}(\mathrm{x}, \mathrm{x}) \mathcal{N}(\mathrm{y}, \mathrm{y})$
Also, iv) for all $\mathrm{x}_{0} \in \mathrm{X}$ the following conditions are equivalent:
a) $\quad \mathcal{N}\left(x_{0}, x_{0}\right)=0$
b) $\quad \mathcal{N}\left(x, x_{0}\right)=0, \forall \mathrm{x} \in \mathrm{X}$
c) $\mathrm{f}\left(x_{0}\right)=0, \forall \mathrm{f} \in \mathcal{H}$

Theorem 2.4. Let be a Hilbert space with a reproducing kernel $\mathcal{N}(\mathrm{x}, \mathrm{y})$, the $\mathcal{N}$ is uniquely determined by $\mathcal{H}$.

The proofs of above results can be seen in [4].

\subsection{Krein Spaces}

Definition 2.5. A topological space with an internal product $(\Re,[r, r])$ and a fundamental decomposition $\mathbb{R}=\mathbb{R}^{+}[+] \mathbb{R}^{-}$is called a Krein space, if the topology on the orthogonal sum coincides with the original topology on the space and the subspaces $\left(\Re^{+},\left[{ }^{\circ}\right]\right),\left(\Re^{-},-\left[r^{\circ}\right]\right)$ are Hilbert spaces.

Note that if a Krein space $\$$ admits a decomposition $\Re=\Re^{+}[\ddot{+}] \Re^{-}$, we can define an internal product $(\mathrm{k}, \mathrm{h}):=\left[\mathrm{k}^{+}, \mathrm{h}^{+}\right]-\left[\mathrm{k}^{-}, \mathrm{h}^{-}\right]$. In addition, there exist projections $\mathrm{P}^{+}$and $\mathrm{P}^{-}$associated to the spaces $\Omega^{+}$and $\Re^{-}$respectively, such that $\left(\mathrm{P}^{ \pm}\right)^{2}=\mathrm{P}^{ \pm}$and $\left[\mathrm{P}^{ \pm} \mathrm{k}, \mathrm{h}\right]=[\mathrm{k}, \mathrm{P} \pm \mathrm{h}]$ for all $\mathrm{k}, \mathrm{h} \in \mathbb{\Re}$. The operator $\mathrm{J}: \Re \rightarrow \Re$ defined by $\mathrm{J}:=\mathrm{P}^{+}-\mathrm{P}^{-}$is called fundamental symmetry. Observe that $\mathrm{J}^{2}=\mathrm{I}_{\mathfrak{\Omega}}$, also

$$
\begin{gathered}
{[\mathrm{k}, \mathrm{h}]_{\mathrm{J}} \mathrm{x}=[\mathrm{Jk}, \mathrm{h}]=(\mathrm{k}, \mathrm{h}) \text { and }} \\
\|\mathrm{k}\|_{\mathrm{J}}:=\sqrt{[\mathrm{k}, \mathrm{k}]_{\mathrm{J}}} \quad \mathrm{k}, \mathrm{h} \in \mathbb{\Omega}
\end{gathered}
$$

The product $\left[{ }^{\circ} \cdot\right]_{\mathrm{J}}$ is called J-internal product and $\left(\Re,\left[{ }^{\circ}\right]_{\mathrm{J}}\right)$ is used to study the linear operators acting on the Krein space $(\Omega,[, \cdot])$. For further information on Krein spaces, we refer to [15] and [16].

Definition 2.6. If $\left(\Re,[\because,]_{\Re}\right)$ and $\left(\varpi,[\because]_{\varpi}\right)$ are Krein spaces with fundamental symmetries $I_{\Omega}$ and Јळ respectively, then 
Observe that the adjoint operator $\mathrm{T}^{*}$ of an operator $\mathrm{T}: \Re \rightarrow \Re$ is given in natural form and the equality $[\mathrm{Tk}, \mathrm{h}]=\left[\mathrm{k}, \mathrm{T}^{*} \mathrm{~h}\right]$ is valid. More precisely if we consider the internal J-product on the Hilbert space $\left(\Re,[\because,]_{j}\right)$ we denoted the adjoint by $\mathrm{T}^{[*]}$ where is the fundamental symmetry of the Krein space.

Definition 2.7. Let be a linear operator with dense

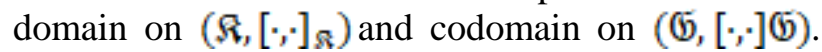
The adjoint operator $\mathrm{T}^{*}$ is defined by

$$
[\mathrm{Tk}, \mathrm{h}]_{\mathscr{W}}=\left[\mathrm{k}, \mathrm{h}^{*}\right]_{\mathbb{K}}, \forall \mathrm{k} \in \operatorname{dom},
$$

where $\quad \mathrm{T}^{*} \mathrm{~h}:=$ is given by

$$
[\mathrm{Tk}, \mathrm{h}]_{\emptyset}=\left[\mathrm{k}, \mathrm{T}^{*} \mathrm{~h}\right]_{\mathbb{R}},
$$

for all $k \in \operatorname{dom}(\mathrm{T})$ and $\mathrm{h} \in \operatorname{dom}\left(\mathrm{T}^{*}\right)$.

The adjoint operator $\mathrm{T}^{*}$ can be expressed through the adjoint $\mathrm{T}^{[\mathrm{k}]}$ which is called $\mathrm{J}$-adjoint and satisfy

$$
[\mathrm{Tk}, \mathrm{g}]_{\mathrm{I} \sigma}=\left[\mathrm{k}, \mathrm{T}^{[*]} \mathrm{g}\right]_{\mathrm{I} \kappa}
$$

for all $\mathrm{k} \in \operatorname{dom}(\mathrm{T})$ and $\mathrm{g} \in \operatorname{dom}\left(\mathrm{T}^{[\mathrm{k}]}\right)$. Moreover [16], we have the equality

$$
\mathrm{T}^{*}=\mathrm{J}_{\mathbb{R}} \mathrm{T}^{[*]} \mathrm{J}_{\Theta}
$$

A linear operator $\mathrm{T}$ is called self-adjoined if $\mathrm{T}=\mathrm{T}^{*}$, and $\mathrm{J}$-self adjoint if $\mathrm{T}=\mathrm{T}^{[\mathrm{f}]}$. In addition, a linear operator $\mathrm{T}$ is invertible if its range and domain are the entire space. The following result can be seen in [14].

Lemma 2.8. Let $(\Omega,[r, r])$ be a Krein space with associated fundamental symmetry $\mathrm{I}$ and $\mathrm{P}$ an orthogonal projection commuting with J, then both $\mathrm{P} \Re$ and $(\mathrm{I}-\mathrm{P}) \Re$ are Krein spaces with fundamental symmetries P J and $(\mathrm{I}-\mathrm{P}) \mathrm{J}$ respectively.

\section{Problem Solution}

The reproducing kernel for a Kerin spaces is defined from the kernels of the defined subspaces that form the associated Hilbert space.

\subsection{Existence of Kernel for Krein Spaces}

Proposition 3.1. Let $(\pi,[\%])$ be a Krein space of complex-valued functions on a set $\mathrm{X}$. If $\bar{K}$ admits a decomposition $\mathfrak{K}:=\mathfrak{K}^{+}[\dot{+}] \mathfrak{K}^{-}$with reproducing kernels $\mathcal{N}^{+} \mathrm{X} \times \mathrm{X} \rightarrow \mathbb{C}$ and $\mathcal{N}^{-}: \mathrm{X} \times \mathrm{X} \rightarrow \mathbb{C}$ for the Hilbert spaces $\left(\Re^{+},\left[{ }^{*},\right)\right.$ and $\left(\Re^{-},-\left[r^{*}\right]\right)$ respectively, then $\mathcal{N}^{+}+\mathcal{N}^{-}$is a reproducing kernel for the Hilbert space $\left(\mathbb{K},[;]_{\mathrm{J}}\right)$ associated to the fundamental symmetry J.

Proof. For each function $\mathrm{k}$ in $\boldsymbol{\mathbb { N }}$, there exist functions $\mathrm{k}^{+}, \mathrm{k}^{-}$in the spaces $\Re^{+}$and $\Re^{-}$ respectively, such $\mathrm{k}=\mathrm{k}^{+}+\mathrm{k}^{-}$and $\Re^{+}[\perp] \Re^{-}$. Suppose that $\mathcal{N}^{+}$is a reproducing kernel for $\mathfrak{K}^{+}$and that $\mathcal{N}^{-}$is a reproducing kernel for $\mathbb{K}^{-}$, then for each $y \in \mathrm{X}, \mathcal{N}_{\mathrm{y}}^{+}$and $\mathcal{N}_{\mathrm{y}}^{-}$are elements in $\mathfrak{K}^{+}$and $\mathfrak{K}^{-}$ respectively. From which we concluded that $\left(\mathcal{N}_{\mathrm{y}}^{+}+\mathcal{N}_{\mathrm{y}}^{-}\right) \quad$ given by $\left(\mathcal{N}_{\mathrm{y}}^{+}+\mathcal{N}_{\mathrm{y}}^{-}\right)(\mathrm{x})=\mathcal{N}_{\mathrm{y}}^{+}(\mathrm{x})+\mathcal{N}_{\mathrm{y}}^{-}(\mathrm{x})$ belong to $\mathfrak{K}$. Also, the reproducing property is fulfilled for $\mathcal{N}^{+}$ and $\mathcal{N}^{-}$in $\left(\mathscr{K}^{+},\left[{ }^{,},\right]\right)$and $\left(\Re^{-},-\left[{ }^{,},\right]\right)$respectively. Therefore, for any $g \in \mathscr{K}^{+}, \mathrm{h} \in \mathfrak{K}^{-}$and for each $\mathrm{x} \in \mathrm{X}$ we have that $\mathrm{g}(\mathrm{x})=\left[\mathrm{g}, \mathcal{N}_{\mathbf{x}}^{+}\right]$and $\mathrm{h}(\mathrm{x})=-\left[\mathrm{h}, \mathcal{N}_{\mathrm{x}}^{-}\right]$. Hence, for each $\mathrm{k} \in \mathfrak{K}$ with $\mathrm{k}=\mathrm{k}^{+}+\mathrm{k}^{-}$and any $\mathrm{x} \in \mathrm{X}$

$$
\begin{aligned}
\mathrm{k}(\mathrm{x}) & =\mathrm{k}^{+}(\mathrm{x})+\mathrm{k}^{-}(\mathrm{x}) \\
& =\left[\mathrm{k}^{+}+\mathrm{k}^{-}, \mathcal{N}_{\mathrm{x}}^{+}-\mathcal{N}_{\mathrm{x}}^{-}\right]
\end{aligned}
$$




$$
\begin{aligned}
& =\left[\mathrm{k}, \mathrm{J}^{2}\left(\mathcal{N}_{\mathrm{x}}^{+}-\mathcal{N}_{\mathrm{x}}^{-}\right)\right] \\
& =\left[\mathrm{k},\left(\mathcal{N}_{\mathrm{x}}^{+}+\mathcal{N}_{\mathrm{x}}^{-}\right)\right]_{J}
\end{aligned}
$$

Consequently $\mathrm{k}(\mathrm{x})=\left[\mathrm{k},\left(\mathcal{N}_{\mathrm{x}}^{+}+\mathcal{N}_{\mathrm{x}}^{-}\right)\right]_{J}$ and then $\mathcal{N}_{\mathrm{x}}^{+}+\mathcal{N}_{\mathrm{x}}^{-}$is a reproducing kernel for $\left(\Re,[;]_{J}\right)$. Observe that, in the above reasoning, $\mathrm{k}(\mathrm{x})=\left[\mathrm{k}, \mathfrak{N}_{\mathrm{x}}^{+}-\mathfrak{Y}_{\mathrm{x}}^{-}\right]$define a reproducing kernel in the following form.

Definition 3.2. Let $(\Re,[;])$ be a Krein space of complex-valued functions on a set $\mathrm{X}$. The function $\mathfrak{N}: \mathrm{X} \times \mathrm{X} \rightarrow \mathbb{C}$ is said to be a reproducing kernel for $(G,[;])$ if the following conditions are true:

i) For each $y \in X$ the function $\mathfrak{N}_{\mathrm{y}}$ defined by $\Re_{y}(\mathrm{x})=\mathfrak{N}(\mathrm{x}, \mathrm{y})$ belong to $\mathfrak{K}$.

ii) For any $\mathrm{x} \in \mathrm{X}$ and $\mathrm{k} \in \mathfrak{K}$, the reproducing property is fulfilled for $\mathrm{k}(\mathrm{x})=\left[\mathrm{k}, \mathfrak{N}_{\mathrm{x}}\right]$.

We can observe that the above definition generalizes the notion of kernel given in the second section for a Hilbert space $\boldsymbol{\xi}$, taking the fundamental symmetry as the identity operator.

Example 3.3. Consider $\ell_{2}(\mathbb{N})$ with the standard

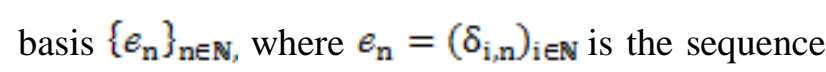
whose $\mathrm{n}-$ th term is 1 and the other terms are zero.

Now, let $\mathfrak{K}_{2}$ be the class of functions $k: \mathbb{N} \rightarrow \mathbb{C}$ such that $\mathrm{k}=\left(\mathrm{k}_{\mathrm{i}}\right)_{\mathrm{i} \in \mathbb{N}} \in \ell_{2}(\mathbb{N}) \quad$ and $\mathrm{k}(\mathrm{i}):=\mathrm{k}_{\mathrm{i}}, \mathbf{i} \in \mathbb{N}$. If we take the internal product given by $\left(k, g \in \Re_{2}(\mathbb{N})\right.$ ) $[\mathrm{k}, \mathrm{g}]_{\aleph^{2}}=\sum_{\mathrm{i} \in \mathbb{N}}(-1)^{\mathrm{i}} \mathrm{k}_{\mathrm{i}} \overline{\mathrm{g}}_{\mathrm{u}}$

Then $\left(\Re_{2}(\mathbb{N}),[; \cdot]_{\mathbb{K}_{2}}\right)$ is a Krein space with fundamental

decomposition

$\mathfrak{K}_{2}(\mathbb{N})=\Re_{2}^{+}[+] \Re_{2}^{-}(\mathbb{N})$, where

$$
\begin{gathered}
\Re_{2}^{+}(\mathbb{N})=\overline{\operatorname{span}\left\{e_{2 \mathrm{n}}\right\}_{\mathrm{n} \in \mathbb{N}}} \text { and } \\
\Re_{2}^{-}(\mathbb{N})=\overline{\operatorname{span}\left\{e_{2 \mathrm{n}-1}\right\}_{\mathrm{n} \in \mathbb{N}}}
\end{gathered}
$$

Thus, each $\mathrm{k} \in \mathbb{K}$ can be written in the form

$$
k=\sum_{i \in \mathbb{N}} k_{2 i} e_{2 i}+\sum_{i \in \mathbb{N}} k_{2 i-1} e_{2 i-1}
$$

Also, for the fundamental symmetry $J_{\mathbb{N}}: \Re_{2}(\mathbb{N}) \rightarrow \Re_{2}(\mathbb{N})$ we have that $\mathrm{J}_{\mathbb{k}}(\mathrm{k})=\left((-1)^{\mathrm{i}} \mathrm{k}_{\mathrm{i}}\right)_{\mathrm{i} \in \mathbb{N}} \cdot$ That is,

$$
\mathrm{J}_{\mathbb{N}} \mathrm{k}=\sum_{\mathrm{i} \in \mathbb{N}} \mathrm{k}_{2 \mathrm{i}} \mathrm{e}_{2 \mathrm{i}}+\sum_{\mathrm{i} \in \mathbb{N}} \mathrm{k}_{2 \mathrm{i}-1} \mathrm{e}_{2 \mathrm{i}-1}
$$

Finally, if $\mathfrak{\Re}^{2+}: \mathrm{X} \times \mathrm{X} \rightarrow \mathbb{C}$ and $\mathfrak{N}^{2-}: \mathrm{X} \times \mathrm{X} \rightarrow \mathbb{C}$ is defined as:

$$
\mathfrak{N}^{2+}(\mathrm{i}, \mathrm{n})=\left\{\begin{array}{cl}
\delta_{\mathrm{i}, \mathrm{n}} & \text { if } \mathrm{n} \text { is even } \\
0 & \text { if } \mathrm{n} \text { is odd }
\end{array}\right.
$$

$\Re^{2-}(\mathrm{i}, \mathrm{n})=\left\{\begin{array}{cc}0 & \text { if } \mathrm{n} \text { is even } \\ \delta_{\mathrm{i}, \mathrm{n}} & \text { if } \mathrm{n} \text { is odd }\end{array}\right.$

Then, both $\Re^{2+}$ and $\mathfrak{\Re}^{2-}$ functions are reproducing kernels for the Hilbert spaces $\left(\mathfrak{K}^{2+}(\mathbb{N}),[;]_{\Re_{2}}\right)$ and $\left(\mathfrak{K}^{2-}(\mathbb{N}),-[\because \cdot]_{\mathbb{K}_{2}}\right)$, respectively. For each $n \in \mathbb{N}$, the function $\mathfrak{N}_{\mathrm{n}}^{2 \pm}$ defined by $\mathfrak{N}_{\mathrm{n}}^{2 \pm}(\mathrm{i}) \mathrm{x}=\mathfrak{N}^{2 \pm}(\mathrm{i}, \mathrm{n})$ belong to $\Re^{2 \pm}(\mathbb{N})$ and satisfy (for $\mathrm{n}$ odd and even)

$$
\sum_{i \in \mathbb{N}}\left|\Re_{n}^{2+}(i)\right|^{2}=\sum_{i \in \mathbb{N}}\left|\delta_{i, n}\right|^{2}=\left|\delta_{n, n}\right|^{2}=1<\infty,
$$


and zero in the other cases. In addition, the reproducing property is verified immediately for $\mathrm{n}$ even and $\mathrm{k} \in \mathbb{K}_{2}^{+}(\mathbb{N})$, because

$$
\begin{aligned}
{\left[k_{,} \mathfrak{N}_{\mathrm{n}}^{2+}\right]_{\mathbb{N}^{2}}=} & \sum_{\mathrm{i} \in \mathbb{N}}(-1)^{\mathrm{i}} \mathrm{k}_{\mathrm{i}} \overline{\mathfrak{H}_{\mathrm{n}}^{2}(\mathrm{l})}=(-1)^{\mathrm{n}} \mathrm{k}_{\mathrm{n}} \delta_{\mathrm{n}, \mathrm{n}} \\
& =\mathrm{k}_{\mathrm{n}}=\mathrm{k}(\mathrm{n})
\end{aligned}
$$

and $\left[k, \Re_{\mathrm{n}}^{2+}\right]_{\mathbb{\aleph}^{2}}=0=\mathrm{k}(\mathrm{n})$, in case odd. In a similar form we can prove this property for the kernel $\Re_{\mathrm{n}}^{2-}$.

Finally, the function $\mathfrak{H}^{2} x=\mathfrak{N}^{2+}+\mathfrak{N}^{2-}$ is a reproducing kernel for the Hilbert space $\left(\Re_{2}(\mathbb{N}),[\because,]_{I R}\right)$ according the Proposition 3.1, where for each $n \in \mathbb{N}$, we can deduce that $\Re_{\mathrm{n}}^{2}=\left(\delta_{\mathrm{i}}, \mathrm{n}\right)_{\mathrm{i} \in \mathbb{N}} \cdot$ Indeed,

$$
\begin{aligned}
{\left[\mathrm{k}_{,} \mathfrak{k}_{\mathrm{n}}^{2+}\right]_{\mathrm{I} \Omega} } & =\sum_{\mathrm{i} \in \mathbb{N}}(-1)^{\mathrm{i}}(-1)^{\mathrm{i}} \mathrm{k}_{\mathrm{i}} \overline{\Re_{\mathrm{n}}^{2}(\mathrm{I})}=\mathrm{k}_{\mathrm{n}} \delta_{\mathrm{n}, \mathrm{n}} \\
& =\mathrm{k}_{\mathrm{n}}=\mathrm{k}(\mathrm{n})
\end{aligned}
$$

In consequence $\mathfrak{R}^{2}$ is a reproducing kernel for the Krein space $\left(\Re_{2}(\mathbb{N}),[\because \cdot]_{\mathbb{R}_{2}}\right)$.

\subsection{Kernel for Krein Spaces}

In this section we prove some useful results to establish relations between reproducing kernel for the Krein spaces and the orthogonal projectors [14].

Lemma 3.4. Let $\left(\pi,\left[{ }^{\circ}\right]\right)$ be a Krein space of complex-valued functions on a non-empty set $\mathrm{X}$ with fundamental symmetry $\mathrm{I}$ and $\mathrm{P}$ an orthogonal projection commuting with J. If $\mathfrak{N}: \mathrm{X} \times \mathrm{X} \rightarrow \mathbb{C}$ is a reproducing kernel for $(\Re,[r])$, then $P \Re$ is a reproducing kernel for $\mathrm{P}$ K.

Proof. If $\mathfrak{\Re}$ is a reproducing kernel for $\mathfrak{K}$ then, for each $y \in X \Re_{y}$ belong to $\Re$ and hence $P \Re_{y}$ belong to $P$ F for all $y \in X$. On the other hand, if $x \in X$ and $k \in K$ then $k(x)=\left[k, \Re_{x}\right]$. Also, if $g=P k \in P \varsubsetneqq$

then $\mathrm{g}$ belong to $\mathscr{K}$. Thus, $\mathrm{P}$ is a projection in $\mathscr{K}$ and $\mathrm{g}(\mathrm{x})=\left[\mathrm{Pk}, \Re_{\mathrm{x}}\right]=\left[\mathrm{Pk}, \mathrm{P} \Re_{\mathrm{x}}\right]=\left[\mathrm{g}, \mathrm{P} \Re_{\mathrm{x}}\right]$. Hence, Pश satisfies the reproducing property and then is a reproducing kernel for P\$.

Proposition 3.5. If $(\xi,[\because])$ is a Krein space of complex-valued functions on $\mathrm{X}$ with a fundamental symmetry $I$, then the following are equivalent:

i) $\mathfrak{N}$ is a reproducing kernel for the Krein space

$$
\text { (अ, }[; \cdot])
$$

ii) $\mathrm{I} \mathfrak{R}$ is a reproducing kernel for the Hilbert space

\section{$\left(\Re_{,}[\because,]_{J}\right)$}

Proof. Let $\mathrm{X}$ be an element of $\mathrm{X}$, then $\Re_{\mathrm{x}} \in \mathfrak{K}$ if and only if $J \Re_{\mathrm{x}} \in \mathfrak{\Re}$. Since $J$ is an operator acting from into itself and satisfy the equality $\mathrm{J}^{2}=\mathrm{Id}$, this property implies that $\left[\mathrm{k}, \mathfrak{\Re}_{\mathrm{z}}\right]=\left[\mathrm{k}, \mathfrak{J}_{\mathrm{k}}\right]_{F}$. Therefore, the equivalence of kernel is proved because by hypotheses, for each $\mathrm{k} \in \mathfrak{\Re}$, 
$\mathrm{k}(\mathrm{x})=\left[\mathrm{k}, \Re_{\mathrm{x}}\right]=\left[\mathrm{k}, \mathfrak{I}_{\mathrm{x}}\right]_{J}$

The following result generalizes the Proposition 2.3 given for Hilbert spaces.

Proposition 3.6. Let $(\mathfrak{G},[; \cdot])$ be a Krein space of complex-valued functions on a set $\mathrm{X}$ and let $\Re: X \rightarrow \mathbb{C}$ be a reproducing kernel. Then, for each $\mathrm{x}$ and $\mathrm{y}$ in $\mathrm{X}, \mathfrak{\Re}$ satisfies the following properties:
i) $\quad \operatorname{IN}(\mathrm{x}, \mathrm{x}) \geq 0$
ii) $\mathfrak{N}(\mathrm{x}, \mathrm{y})=\overline{\mathcal{N}(\mathrm{y}, \mathrm{x})}$
iii) $|\mathfrak{i}(\mathrm{x}, \mathrm{y})|^{2} \leq(\mathrm{J})(\mathrm{x}, \mathrm{x})(\mathrm{J} \Re)(\mathrm{y}, \mathrm{y})$

Also,

iv) For each $\mathrm{x}_{0} \in \mathrm{X}$, the following conditions are equivalent:

a) $\mathfrak{N}\left(\mathrm{x}_{0}, \mathrm{x}_{0}\right)=0$

b) $\mathfrak{i}\left(\mathrm{x}, \mathrm{x}_{0}\right)=0, \forall \mathrm{x} \in \mathrm{X}$

c) $\mathrm{k}\left(\mathrm{x}_{0}\right)=0, \forall \mathrm{k} \in \mathfrak{k}$.

Proof. Let $\mathfrak{\Re}$ be a reproducing kernel for a Krein space $(\xi,[\because])$ and $x, y \in X$. The properties ii) and iv) are clear. Also, according to Proposition 3.5,

$$
\mathrm{J}(\mathrm{x}, \mathrm{x})=\left[\mathrm{J} \Re_{w} \mathfrak{J}_{\mathrm{z}}\right]_{J}=\left\|\Re_{\mathrm{z}}\right\| \mathrm{J} \geq 0
$$

Finally, by Cauchy Schwarz's inequality, it follows iv),

$$
|\mathcal{N}(\mathrm{x}, \mathrm{y})|^{2}=\left\|\left[\Re_{\mathrm{y}}, \Re_{\mathrm{x}}\right] \mid \leq\right\| \Re_{\mathrm{x}}\left\|_{J}^{2}\right\| \Re_{\mathrm{y}} \|_{J}^{2}
$$

$$
\begin{aligned}
& =\left\|\Re_{\mathrm{x}}\right\|_{J}^{2}\left\|\Re_{\mathrm{y}}\right\|_{J}^{2} \\
& =\left[\Re_{\mathrm{x}} \mathrm{J} \Re_{\mathrm{x}}\right]\left[\Re_{\mathrm{y}} \mathrm{J} \Re_{\mathrm{y}}\right] \\
& =\left(\mathrm{J} \Re_{\mathrm{x}}\right)(\mathrm{x})\left(\mathrm{J} \Re_{\mathrm{y}}\right)(\mathrm{y}) \\
& =(\mathrm{J} \mathcal{N})(\mathrm{x}, \mathrm{x})(\mathrm{J} \mathcal{N})(\mathrm{y}, \mathrm{y})
\end{aligned}
$$

The Example 3.3 exhibits relationship among the reproducing kernels of $\mathfrak{k}, \mathfrak{K}^{+}$and $\mathfrak{K}^{-}$. The relationship among these kernels is proved in the following theorem.

Theorem 3.7. Let $\left(\sigma_{,}\left[r^{*}\right]\right)$ be a Krein space of complex-valued functions on a set $\mathrm{X}$ with a decomposition $\Re=\Re^{+}[\dot{+}] \Re^{-}$. If $\Re: X \times X \rightarrow \mathbb{C}$ is a reproducing kernel for $\Re$, then there exist reproducing kernels $\mathfrak{\Re}^{+}, \mathfrak{N}^{-}$for Hilbert spaces $\mathfrak{K}^{+}$ and $\mathfrak{K}^{-}$respectively, such $\mathfrak{\Re}=\mathfrak{R}^{+}-\mathfrak{N}^{-}$.

Proof. Let $\mathfrak{\Re}$ be a reproducing kernel for the Krein space $\mathscr{K}=\Re^{+}[\dot{+}] \Re^{-}$and $y$ an element of $\mathrm{X}$, then the function $\mathfrak{N}_{\mathrm{y}}$ defined by $\mathfrak{N}_{\mathrm{y}}(\mathrm{x})=\mathfrak{N}(\mathrm{x}, \mathrm{y})$ belong to $\mathfrak{K}$ and there exist $\mathfrak{N}_{\mathrm{y}}^{1}, \mathfrak{N}_{\mathrm{y}}^{2}$ in the Hilbert spaces $\mathfrak{K}^{+}$and $\mathfrak{K}^{-}$respectively, such $\Re_{\mathrm{y}}=\mathfrak{N}_{\mathrm{y}}^{1}+\mathfrak{N}_{\mathrm{y}}^{2}$. Now it must be proved that the functions $\Re^{+}: \mathrm{X} \times \mathrm{X} \rightarrow \mathbb{C}$ and $\mathfrak{N}^{-}: \mathrm{X} \times \mathrm{X} \rightarrow \mathbb{C}$ given by

$\Re^{+}(\mathrm{x}, \mathrm{y}):=\mathfrak{N}_{\mathrm{y}}^{1}(\mathrm{x})$ and $\mathfrak{H}^{-}(\mathrm{x}, \mathrm{y}):=-\mathfrak{N}_{\mathrm{y}}^{2}(\mathrm{x})$

are reproducing kernels for the spaces $\left(\kappa^{+},\left[{ }^{*}\right]\right)$ and $\left(a^{-},-\left[r^{,}\right]\right)$respectively. Indeed, for each $y \in X$ the 
functions $\Re_{\mathrm{y}}^{+}$and $\mathfrak{N}_{\mathrm{y}}^{-}$defined by $\mathfrak{N}_{\mathrm{y}}^{+}(\mathrm{x}):=\mathfrak{H}^{+}(\mathrm{x}, \mathrm{y})$ and $\mathfrak{N}_{\mathrm{y}}^{-}(\mathrm{x}):=\mathfrak{H}^{-}(\mathrm{x}, \mathrm{y})$ meet the following

$\Re_{\mathrm{y}}^{+}(\mathrm{x})=\mathfrak{N}_{\mathrm{y}}^{1}(\mathrm{x})$ and $\mathfrak{R}_{\mathrm{y}}^{-}(\mathrm{x})=-\mathfrak{N}_{\mathrm{y}}^{2}(\mathrm{x}), \forall \mathrm{x} \in \mathrm{X}$

whereby for each $y \in \mathrm{X}$ the functions $\mathfrak{\Re}_{\mathrm{y}}^{+}$and $\mathfrak{\Re}_{\mathrm{y}}^{-}$ are elements of the Hilbert spaces $\mathfrak{K}^{+}$and $\mathfrak{K}^{-}$ respectively. Moreover, from this it is easily seen that $\mathfrak{R}=\mathfrak{N}^{+}-\mathfrak{N}^{-}$. As well if $\mathrm{h} \in \mathfrak{K}^{+} \subset \mathfrak{K}$ then $\mathrm{h}(\mathrm{x})=\left[\mathrm{h}, \mathfrak{Y}_{\mathrm{x}}\right]$ for each $\mathrm{x} \in \mathrm{X}$ by using the reproducing property of kernel $\mathrm{N}$. Therefore,

$$
\begin{aligned}
\mathrm{h}(\mathrm{x}) & =\left[\mathrm{h}, \mathfrak{N}_{\mathrm{x}}^{+}-\mathfrak{\Re}_{\mathrm{x}}^{-}\right]=\left[\mathrm{h}, \mathfrak{N}_{\mathrm{x}}^{+}\right]-\left[\mathrm{h}, \mathfrak{N}_{\mathrm{x}}^{-}\right] \\
& =\left[\mathrm{h}, \mathfrak{\Re}_{\mathrm{x}}^{+}\right]-0 \\
& =\left[\mathrm{h}, \mathfrak{\Re}_{\mathrm{x}}^{+}\right]
\end{aligned}
$$

Similarly, if $\mathrm{g} \in \mathfrak{K}^{-} \subset \mathfrak{K}$ then $\mathrm{g}(\mathrm{x})=\left[\mathrm{g}, \mathfrak{F}_{\mathrm{x}}\right]$ and

$$
\begin{aligned}
\mathrm{g}(\mathrm{x})=\left[\mathrm{g}, \Re_{\mathrm{x}}^{+}-\mathfrak{N}_{\mathrm{x}}^{-}\right]=\left[\mathrm{g}, \mathfrak{N}_{\mathrm{x}}^{+}\right]-\left[\mathrm{g}, \mathfrak{N}_{\mathrm{x}}^{-}\right] \\
\quad=0-\left[\mathrm{g}, \Re_{\mathrm{x}}^{-}\right] \\
\quad=-\left[\mathrm{g}, \mathfrak{N}_{\mathrm{x}}^{-}\right]
\end{aligned}
$$

Thus, the reproducing properties of reproducing kernels $\mathfrak{N}^{+}$and $\mathfrak{N}^{-}$are proved for the Hilbert spaces $\left(\mathfrak{G}^{+},\left[{ }^{\prime},\right]\right)$ and $\left(\mathfrak{G}^{-},-\left[r^{0}\right]\right)$, respectively.

In other words, Theorem 3.7 guarantees us a natural way to define the nucleus of reproduction for a Krein space. Even with theorem 3.8 we have the implication that said nucleus of reproduction is independent of the fundamental symmetry since any other nucleus is equivalent to that of the previous theorem.
Theorem 3.8. Let $(\Re,[r])$ be a Krein space of complex-valued functions on a set $\mathrm{X}$. The reproducing kernel $\mathfrak{\Re}$ is uniquely determined by the Krein space $\boldsymbol{K}$.

Proof. Suppose that a Krein space $\$$ with fundamental symmetry I has a reproducing kernel $\Re^{1}$. If $\mathfrak{\Re}^{2}$ is another reproducing kernel for $\mathfrak{K}$, by Proposition 3.6 it follows that for all $y \in X$,

$$
\begin{aligned}
\left\|\Re_{\mathrm{y}}^{1}-\Re_{\mathrm{y}}^{2}\right\|_{\mathrm{J}}^{2}=\left[\Re_{\mathrm{y}}^{1}-\Re_{\mathrm{y}}^{2}, \Re_{\mathrm{y}}^{1}-\Re_{\mathrm{y}}^{2}\right]_{\mathrm{J}} \\
\quad=\left[\mathrm{J}\left(\Re_{\mathrm{y}}^{1}-\Re_{\mathrm{y}}^{2}\right), \Re_{\mathrm{y}}^{1}\right]-\left[\mathrm{J}\left(\Re_{\mathrm{y}}^{1}-\Re_{\mathrm{y}}^{2}\right), \Re_{\mathrm{y}}^{2}\right]
\end{aligned}
$$

Additionally, by the reproducing property of $\Re^{1}$ and $\Re^{2}$, we obtain the equalities

$$
\begin{aligned}
\left\|\Re_{y}^{1}-\Re_{y}^{2}\right\|_{\mathrm{I}}^{2} & =J\left(\Re_{y}^{1}-\Re_{y}^{2}\right)(y)-J\left(\Re_{y}^{1}-\Re_{y}^{2}\right)(y) \\
& =0
\end{aligned}
$$

Then, $\mathfrak{N}_{\mathrm{y}}^{1}=\mathfrak{N}_{\mathrm{y}}^{2}$ for each $\mathrm{y} \in \mathrm{X}$. Thus, for any $\mathrm{x}, \mathrm{y} \in \mathrm{X}$,

$\mathfrak{N}^{1}(\mathrm{x}, \mathrm{y})=\mathfrak{\Re}^{2}(\mathrm{x}, \mathrm{y})$

\section{Conclusion}

We describe the kernel of a Krein space as the difference between the kernel of definite positive subspace and the kernel of definite negative subspace. Also, we prove some basic properties of both kernels for Krein spaces and we exhibit that each kernel is uniquely determined by the Krein space given. In consequence, we generalize the notion of reproducing kernel given in Hilbert spaces 
to the context of spaces endowed with indefinite metric.

\section{References:}

[1] Berlinet A and Thomas-Agnan C 2004 Reproducing Kernel Hilbert Spaces in Probability and Statistics vol 22 (Boston: Kluwer Academic Publishers)

[2] Dym H 1989 Contractive Matrix Functions, Reproducing Kernel Hilbert Spaces and Interpolation vol 71 (Washington: Published for the Conference Board of the Mathematical Sciences)

[3] Yao K 1967 Applications of reproducing kernel Hilbert spaces Inf. Control 11 429-444

[4] Zaremba, S. 1907 L biharmonique et une classe remarquable de fonctions harmoniques. Bulletin International de l'Académie des Sciences de Cracovie 147-196

[5] Mercer J 1909 Functions of positive and negative type and their connection with the theory of integral equations Philos. Trans. Royal Soc. A 209 415-446

[6] Bergman S 1950 The Kernel Function and Conformal Mapping vol 5 (Providence: American Mathematical Society)

Bochner S and Gunning R Collected papers. Parts 1, 2, 3, 4. Amer. Math. Sco

[7] Szego G and Po'lya G 1998 Problems and Theorems in Analysis I vol 19 (Heidelberg: Springer-Verlag)

[8] Mohammadi M, Saberi Zafarghandi F, Babolian E and Jvadi S 2018 A local reproducing kernel method accompanied by some different edge improvement techniques: application to the Burgers' equation, Iranian Journal of Science and Technology, Transaction A: Science 42(2), pp. 857-871

[9] Oglic D and Gärtner T 2018 Learning in reproducing kernel Kreïn spaces, 35th International Conference on Machine Learning, ICML 2018 9, pp. 6188-6200

[10] Saha A and Balamurugan P, 2020 Learning with operator-valued kernels in reproducing kernel krein spaces, Advances in Neural Information Processing Systems 2020December

[11]Liu F, Shi L, Huang X, Yang J, Suykens J.A.K., 2021 Analysis of regularized leastsquares in reproducing kernel Kreĭn spaces, Machine Learning 110(6), pp. 1145-1173

[12]Alpay D, Jorgensen P and Volok D, 2014 Relative Reproducing Kernel Hilbert Spaces,
American Mathematical Society vol 14211 3889-3895

[13]Lopatto P and Rochberg R, 2015 SchattenClass Truncated Toeplitz Operators, American Mathematical Society vol 1442 637-649

[14]Esmeral K, Ferrer O and Wagner E 2015 Frames in Krein spaces arising from a nonregular W-metric, Banach J. Math. Anal. 9 116

[15]Escobar G, Esmeral K and Ferrer O 2016 Construction and coupling of frames in Hilbert spaces with W-metrics, Integración 34 81-93

[16]Azizov T and Iokhvidov I 1989 Linear Operators in Spaces with an Indefinite Metric (Chichester: Pure and Applied Mathematics, John Wiley and Sons Ltd)

[17]J. Bognar 1974 Indefinite Inner Product Spaces (New York: Springer-Verlag)

\section{Contribution of Individual Authors to the Creation of a Scientific Article (Ghostwriting Policy) \\ Osmin Ferrer carried out the conceptualization, methodology and supervision. \\ Diego Carrillo was responsible for the writing of the original draft. \\ Arnaldo de la Barrera wrote the corresponding review and editing.}

\section{Creative Commons Attribution License 4.0 (Attribution 4.0 International, CC BY 4.0)}

This article is published under the terms of the Creative Commons Attribution License 4.0 https://creativecommons.org/licenses/by/4.0/deed.en US 